\title{
Moisture Transport for Reaction Enhancement in Fabrics
}

\author{
Phillip Gibson, ${ }^{1}$ Heidi Schreuder-Gibson, ${ }^{1}$ Pearl Yip, ${ }^{1}$ Brendan Denker, ${ }^{1}$ Hamid Benaddi, ${ }^{2}$ \\ Sa Wang, ${ }^{3}$ Lev Bromberg, ${ }^{3}$ and T. Alan Hatton ${ }^{3}$ \\ ${ }^{1}$ U.S. Army Natick Soldier Research, Development and Engineering Center, Natick, MA 01760, USA \\ ${ }^{2}$ Stedfast, Inc., Granby, QC, Canada J2G $3 Y 3$ \\ ${ }^{3}$ Department of Chemical Engineering, Massachusetts Institute of Technology, Cambridge, MA 01239, USA
}

Correspondence should be addressed to Phillip Gibson; phillip.gibson@us.army.mil

Received 8 November 2012; Accepted 29 January 2013

Academic Editor: Sambandam Anandan

Copyright (C) 2013 Phillip Gibson et al. This is an open access article distributed under the Creative Commons Attribution License, which permits unrestricted use, distribution, and reproduction in any medium, provided the original work is properly cited.

\begin{abstract}
The role of water in protective fabrics is critical to comfort and material performance. Excessive perspiration in clothing causes discomfort, and bound water can adversely affect the ability of carbon to adsorb chemicals. Yet the presence of water can also improve the moisture vapor transport of protective polymer films, and is essential for the hydrolytic destruction of nerve agents. Reported here are the findings of wicking and drying experiments conducted on various hydrophilic and hydrophobic cover fabrics that demonstrate the influence of wetting on permeation through fabrics. The influence of water content on reactive polymers capable of degrading nerve agent simulant is also discussed, and the importance of a novel "delivery system" for water to the reactive components through the use of a wicking fabric is introduced.
\end{abstract}

\section{Introduction}

Current chemical protective fabrics rely on layers of activated carbon to adsorb chemical agent vapors. A tightly woven outer hydrophobic and oleophobic shell fabric provides liquid protection and aerosol particle protection.

Another approach is to base protection on a semipermeable or reactive polymer membrane layer, which is permeable to water vapor, but which is also a barrier to chemical agents. The polymer membrane is usually laminated between an outer shell fabric and an inner fabric layer in contact with the skin. The outer fabric layer is treated to be hydrophobic and oleophobic. This allows water (rain), oils, and chemical agents to roll off of the fabric surface. However, the inner layer of these fabrics can be designed to pick up liquid sweat from the skin's surface. The liquid wicking characteristics of this inner laminated "comfort layer" fabric can affect the perceived comfort of the garment and the total amount of sweat vapor capable of being transmitted through the laminated fabric and membrane structure.

The research gap motivating the work presented in this study is the need to more fully understand how water amount and location affect reactive compounds present in fabrics and polymer membranes that are designed to hydrolytically decompose chemicals that are analogs for nerve agents. One such compound is the polymeric supernucleophilic pyridine catalyst called poly(butadiene-co-pyrrolidinopyridine) (polyBPP) [1-3], which functions to completely degrade the nerve agent surrogates, including the compound, diisopropyl fluorophosphate (DFP).

Water interactions are involved in the degradation kinetics of DFP and require that there must be a sufficient amount of water and complete contact between the DFP, water, and catalyst for rapid and complete destruction of the surrogate. The delivery of the liquid water from the interior of the protective fabric to the exterior regions containing the reactive compounds is an important process that has been designed into a layered fabric system comprised of a wicking comfort layer next to the skin, bonded to a polymer membrane capable of degrading DFP, and covered with a protective outer shell fabric that can be modified with polyBPP. Previous work on water interactions with chemical protective polymer membranes has shown that water content of the polymer layer is important for permeation of water and chemical 


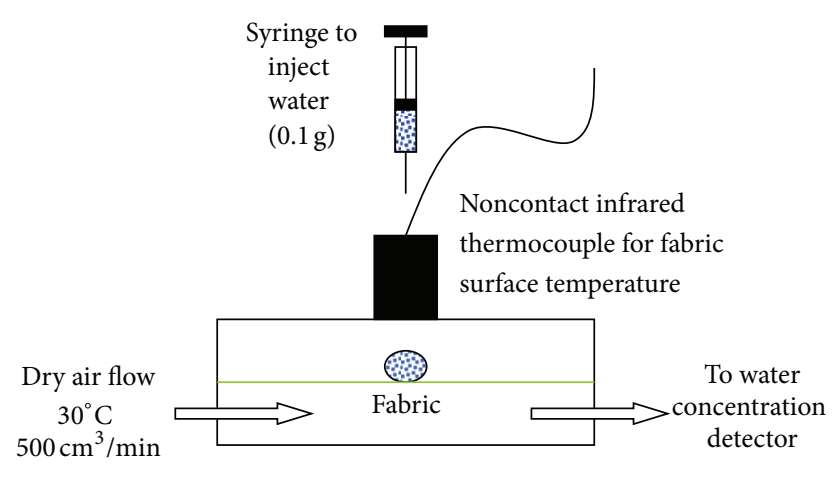

Figure 1: Test cell modified to accommodate an infrared thermocouple viewing fabric surface in the cell.

agent simulants [4-6]. Of particular interest is the increase in efficiency that the wicking comfort layer might contribute to the hydrolytic degradation of DFP within the reactive membrane. This paper focuses on fabric design features that promote efficient water interactions with reactive compounds within layered fabric systems.

\section{Liquid Water Wicking/Transport}

Novel drying experiments were developed to measure drying time by means of vapor flux from spreading liquids in a modified permeation test cell. Previous measurements of this type have been useful in distinguishing the difference in performance of single-layer fabrics that differ in their liquid wicking characteristics [7].

Measurement systems as shown in Figure 1 provide water vapor flux through the fabric as influenced by fabric surface treatments.

Fabrics can be treated to be fully wicking on both sides, partially wicking on one side and hydrophobic on the other side or fully hydrophobic on both sides. Figure 2 shows typical examples of water behavior in a single layer of fabric (hydrophobic on one side, and hydrophilic on the other) and the way that water wets the inner "comfort layer" of an activated carbon chemical protective garment, while it beads up on the outer protective shell fabric layer.

Figure 3 shows typical drying curves for a variety of single-layer fabrics that differ in their hydrophobic surface characteristics on one or both sides. It is clear that gradations of treatment can produce fabrics with transport behavior located anywhere on the spectrum between "fully wicking" and "fully repellent." Modern textile fabrication and finishing technology provides the ability to tailor fabric wicking and repellency to achieve specific performance requirements; however, it is unclear in some design applications what those performance targets should be.

Previous work on the wicking and wetting characteristics of fabrics showed that there are physiological and comfort consequences to the choice of fabric wicking characteristics [7]. The orientation of a fabric with wicking characteristics on one side and hydrophobic characteristics on the other is of particular importance.
Similar experiments on wicking and water transport were performed for a variety of more complicated fabrics. Included were several commercial polymer membrane laminates used in "breathable" rainwear. The behavior of these materials becomes much more complicated, since these are multilayer structures that usually include a solid polymer film that changes its transport behavior depending on how much water is in the polymer structure. The most interesting aspect of these measurements was the resulting observation that many of these materials had poor water vapor transport at low states of hydration but performed very well when exposed to liquid water at the same conditions. The improved performance seemed related to the presence of an inner wicking fabric layer that ensured liquid was transported through the fabric quickly to the polymer layer, where water was able to hydrate the membrane and quickly pass through to the other side.

An example of this increased water vapor transport for polymer membrane laminates exposed to liquid water is shown in Figure 4. A reactive polymer membrane laminate that had poor water vapor transport under steady-state vapor conditions showed improved performance approaching that of a fully wicking glass fiber filter layer (used as a control). The fabric surface temperature can be monitored during the wicking and drying process as another indicator of drying time, since the evaporation of the liquid causes a drop in surface temperature (Figure 4).

The measurement shown in Figure 4 was interesting, because it showed that a reactive polymer membrane laminate performed well at pushing water (sweat) from the skin surface through the wicking comfort layer to the reactive polymer membrane layer, where the water becomes available to aid in chemical reactions that degrade chemical warfare agents, as well as hydrating the membrane to improve water vapor transport. A more controlled study of the specific transport behavior of polymer membrane laminates, as opposed to single-fabric layers, was developed to understand these phenomena.

\section{Materials and Methods}

We chose a single-fabric system to study the effect of differential water and wicking performance of a "comfort layer" on the inner surface of a reactive polymer membrane laminate. The reactive polymer membrane layer is manufactured by Stedfast, Inc., under the trade name of ChemCAT. A knit comfort layer was chosen as the fabric to laminate to the inner surface of the membrane. The knit fabric is engineered to be hydrophilic on one side and hydrophobic on the other side. However, although the hydrophobic side is water repellent to some degree, it is capable of picking up liquid water (sweat) from the skin surface and transporting it through to the hydrophilic side, where it is retained. As a consequence, the hydrophobic side will wick sweat away from the skin and will have a very dry feel, since the liquid is segregated away from the skin surface in the outer hydrophilic layer.

By contrast, if the hydrophilic layer is against the skin, the sweat will be wicked into that layer, stay there against the skin, 


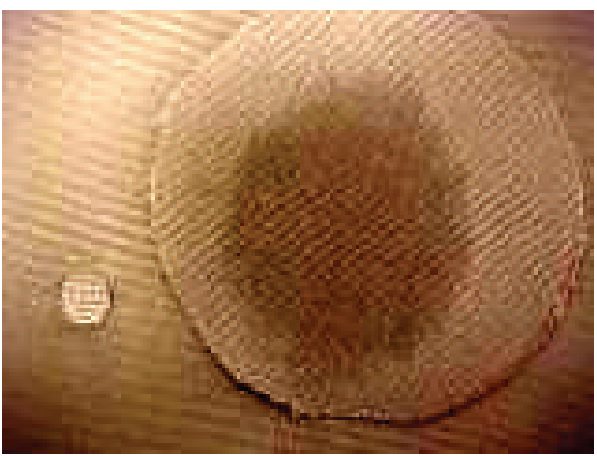

(a)

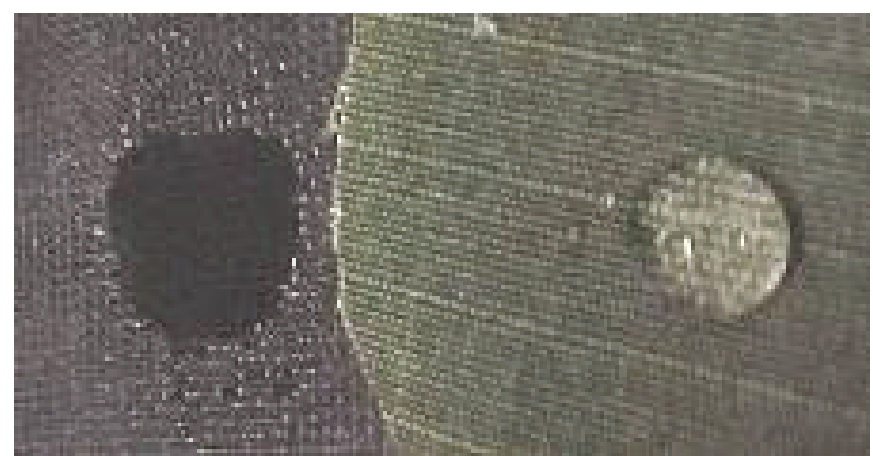

(b)

FIGURE 2: Water on inner and outer surfaces of fabrics for drying experiments. (a) Single-layer fabric with hydrophilic treatment on inner surface and hydrophobic treatment on outer surface. (b) Standard carbon inner liner and outer shell fabric.

TABLE 1: Wicking test conditions.

(i) $0.1 \mathrm{~g}$ of Liquid water applied to top surface of fabric.

(ii) Test temperature of $30^{\circ} \mathrm{C}$.

(iii) Gas flow rates of $2 \mathrm{~L} / \mathrm{min}$ on both sides of fabric.

(iv) Humidity is fractional ( 0.80 is $80 \%$ r.h.).

\begin{tabular}{lcccc}
\hline Humidity on top & Humidity on bottom & Humidity gradient & Mean relative humidity \\
\hline 0.0 & \multicolumn{4}{c}{ Constant humidity conditions } \\
0.4 & 0.0 & N/A & 0.0 \\
0.8 & 0.4 & N/A & 0.40 \\
\hline \multicolumn{7}{c}{ N/A } & 0.80 \\
\hline 0.4 & 0.8 & Humidity gradient conditions & 0.4 \\
0.6 & 0.0 & 0.4 & 0.4 \\
0.8 & 0.2 & 0.4 & 0.6 \\
\hline
\end{tabular}

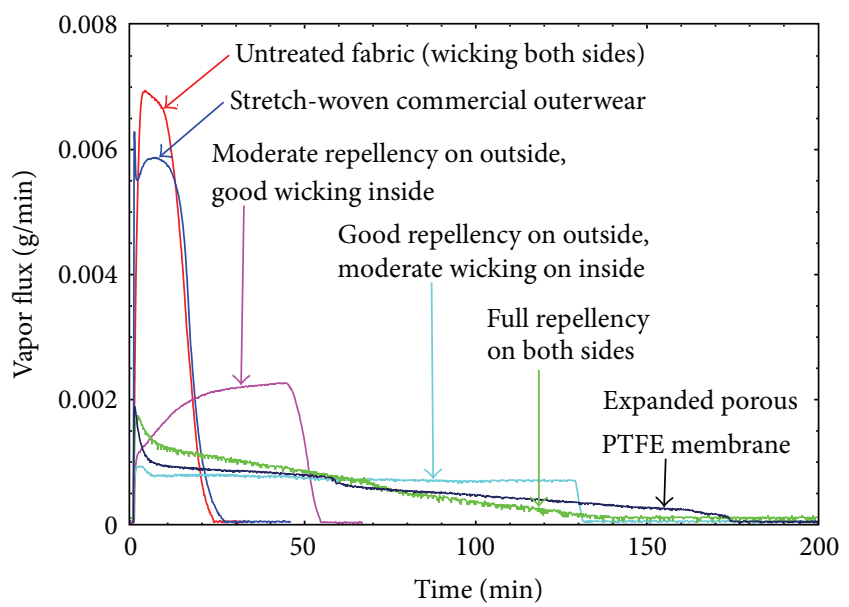

FIGURE 3: Typical drying behavior of fabrics with various wicking treatments on the inner and outer surfaces of a single layer of fabric [7].

and produce a much great sensation of wetness. The outer hydrophobic surface does not become wetted in this case.

Two variants of laminated fabric were produced with this hydrophobic/hydrophilic comfort layer: (1) the hydrophilic layer was oriented toward the skin surface (hydrophobic layer attached to the polymer membrane) and (2) the hydrophobic layer was oriented toward the skin surface (hydrophilic layer attached to the polymer membrane).

We supposed that the consequences of these two fabric orientations would be that, in case (1) where the hydrophilic layer was against the skin, the liquid water would be less available to the polymer membrane layer, since the water would not pass through the hydrophobic layer. For case (2), the hydrophobic fabric would pass water directly to the hydrophilic layer against the polymer membrane, where it would be quickly absorbed, producing a higher hydration state within the reactive polymer, enhancing transport and reactivity.

A wicking glass fiber filter layer was used as a control. This material was a borosilicate glass filter, with no resin binder or hydrophobic or oleophobic treatments. The glass fiber filter had high porosity and low air permeability and held liquid water within the pore spaces of the material with no preferential dependence of wicking through the material thickness. Figure 5 outlines the various material configurations used for the wicking study.

The test system shown in Figure 6 was modified slightly from that shown previously in Figure 1. Equal gas flows were 


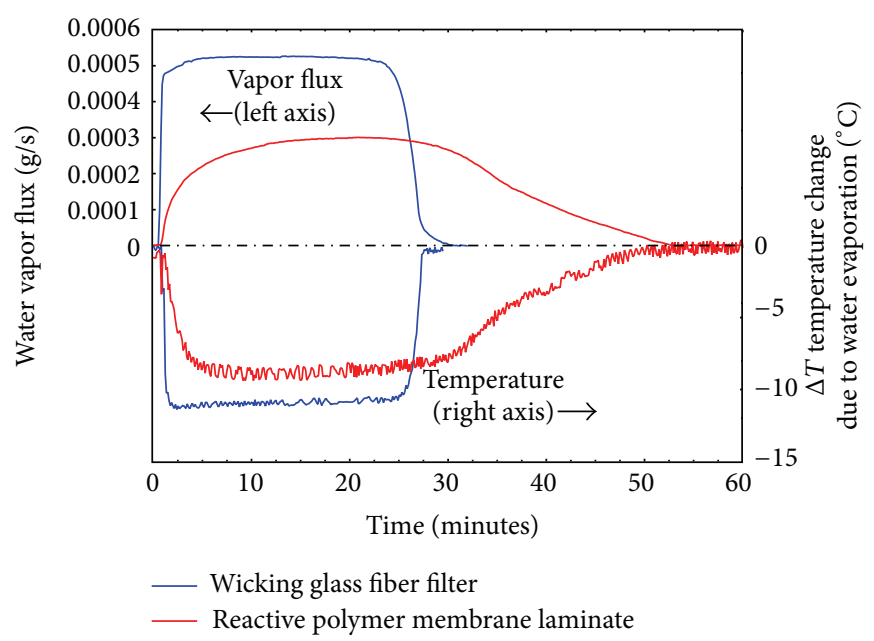

FIGURE 4: Simultaneous vapor flux and temperature drop measurements for a fully wicking layer and a reactive polymer laminate that includes a wicking fabric layer on the inner surface.

\begin{tabular}{ll}
\multicolumn{1}{c}{ Wicking hydrophobic surface } & Differential wicking comfort fabric (single layer) \\
& -If water is applied to hydrophobic side, \\
Wicking hydrophilic surface & water initially beads up, but time or pressure \\
& will cause water to preferentially migrate to \\
& hydrophilic side of fabric. \\
\hline $\begin{array}{l}\text { Fabric laminated to } \\
\text { reactive polymer membrane }\end{array}$ & -If water is applied to hydrophilic side, \\
\hline
\end{tabular}

Material test configurations for liquid water applied to inner surface (body side)

-Fabric laminated to reactive polymer membrane in two orientations

Hydrophilic wicking inner surface

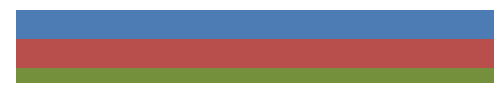

Hydrophobic wicking inner surface

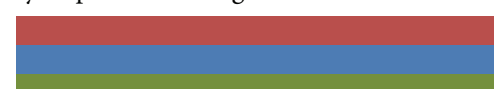

-Liquid water held next to membrane

-Membrane more hydrated

-Inner layer (body side) feels dry

Liquid water equally distributed
Glass fiber filter paper

fully wicking layer
-Liquid water held away from membrane

-Membrane less hydrated

-Inner layer (body side) feels wet

FIgURE 5: Material configurations for liquid water wicking and transport study. used to sweep the top and bottom of the cells, and the humidity and flux was measured from both the top and bottom surfaces of the sample. This allowed the differences between the evaporative losses from the top surface to be compared directly to the water vapor flux that were actually transmitted through the fabric and polymer membrane layers.
The use of this configuration also allowed the sample to be equilibrated to a variety of humidity conditions, some of which included a gradient of humidity from the top to the bottom of the permeation cell (Table 1). This allowed direct comparison of the water vapor flux measured under vapor conditions to that obtained under the conditions where liquid 


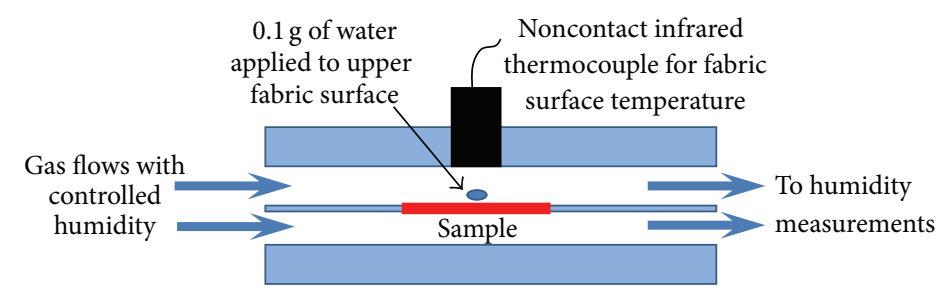

FIGURE 6: Experimental system of Figure 1 modified to add a ventilating top flow and simultaneous measurement of water vapor flux from the top and bottom flow channels of the permeation cell.

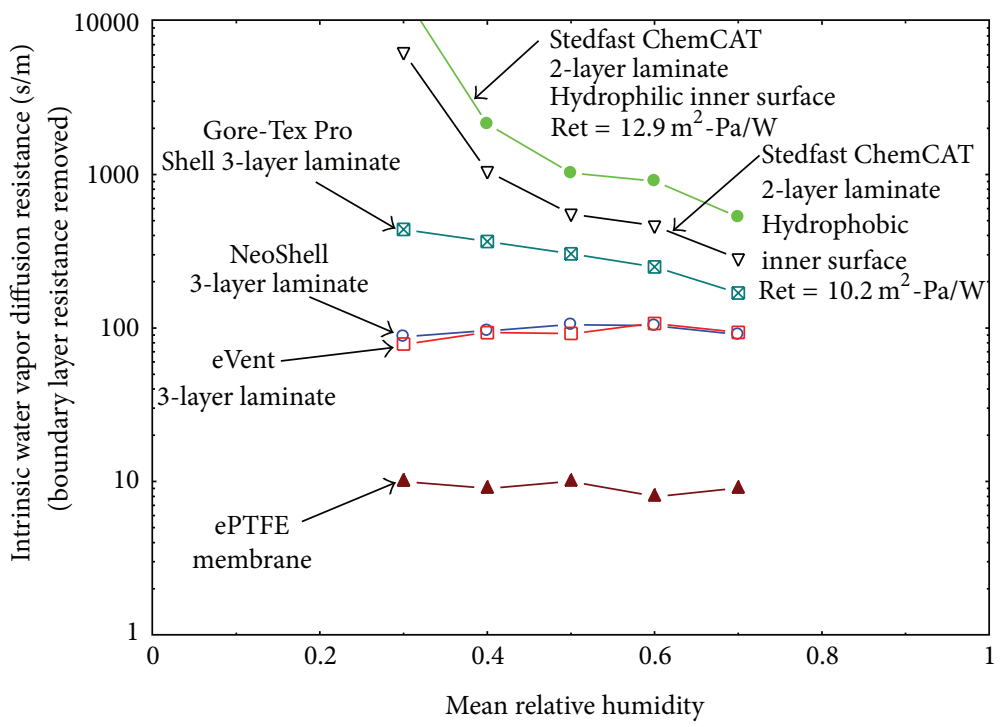

FIGURE 7: Water vapor diffusion resistance of ChemCAT laminates and several reference materials. Measured Ret values (ISO 11092) shown for ChemCAT laminates are consistent with the diffusion resistance values.

sweat is present. Further details on the instrumentation used in the permeation cell are available $[4,7]$.

Table 1 shows the wicking test conditions used for the study.

\section{Results}

The steady-state water vapor permeation results for the two laminates are shown in Figure 7. The results are shown in terms of water vapor diffusion resistance, where lower resistance implies better performance and comfort [4]. "Mean relative humidity" refers to the mean of the humidity on the two sides of the sample and is related to the equilibrium water content in the sample-a higher mean relative humidity implies higher water content in the polymer layer. The results shown in Figure 7 are consistent with many previous studies showing that the state of hydration and equilibrium water content in polymer membranes can have a large effect on the water vapor transport properties [8-16].

Figure 7 shows that the reactive polymer membrane laminates have high resistance to water vapor transport when compared to three high-performance commercial waterproof-breathable membrane laminates and a standard reference material (expanded polytetrafluoroethylene
(ePTFE)). Although there are some differences in the measured properties of the two ChemCAT samples, we believe the differences are probably due to fabrication variations in the membrane and lamination method, rather than due to the orientation of the fabric layer. These commercial materials are similar in construction to the reactive polymer membrane laminates and serve as desirable performance goals for the reactive laminates. The "NeoShell" and the "eVent" laminates are composed of air permeable polymer membrane layers, while the Gore-Tex "Pro Shell" includes a thin solid polymer membrane layer much like the ChemCAT material.

Scanning electron micrographs of the functional polymer layer for each of the comparison materials are shown in Figure 8.

What are the consequences of the differing orientation of the laminated comfort layer upon the water vapor transport behavior of reactive polymer membrane laminates?

A representative case in Figure 9 shows that the reactive membrane laminate with the hydrophobic wicking inner surface has a very different water transport profile than the laminate with the reverse orientation (hydrophobic fabric side against the membrane). When the liquid water is held directly against the membrane in the hydrophilic layer, the water is transported quickly through the laminate, and a higher total amount is transported (area under the curve) 


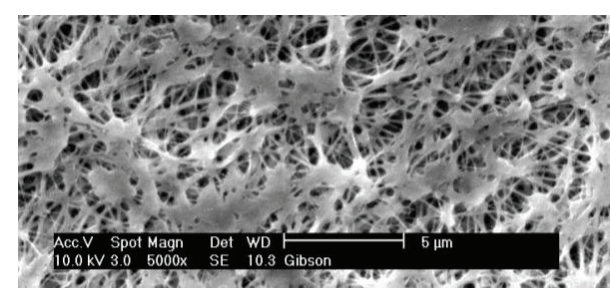

(a) eVent ePTFE film

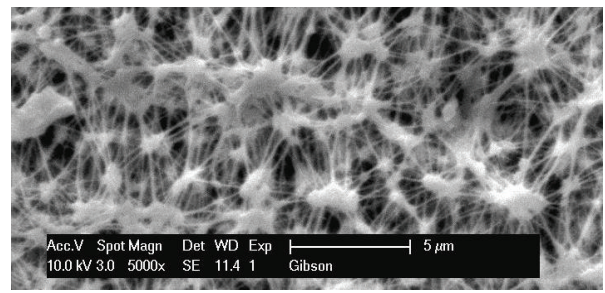

(c) Gore-Tex Membrane (uncoated side: ePTFE )

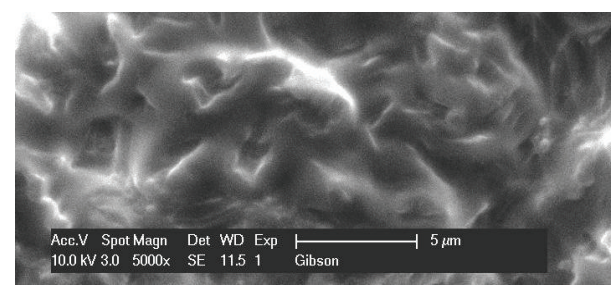

(b) Gore-Tex Membrane (coated side: continuous polymer film)

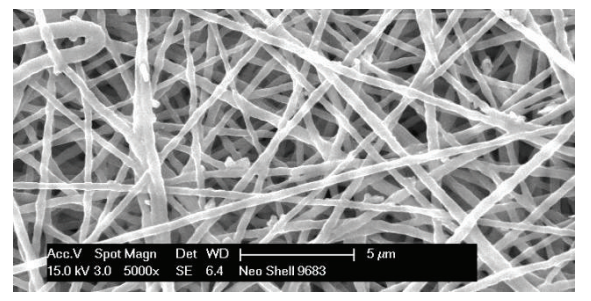

(d) NeoShell Electrospun Polyurethane Membrane

FIGURE 8: Scanning electron microscope images of typical membranes used in waterproof breathable fabric laminates. Images are of the membrane layer only (bonded supporting and protective textile layers: not shown).

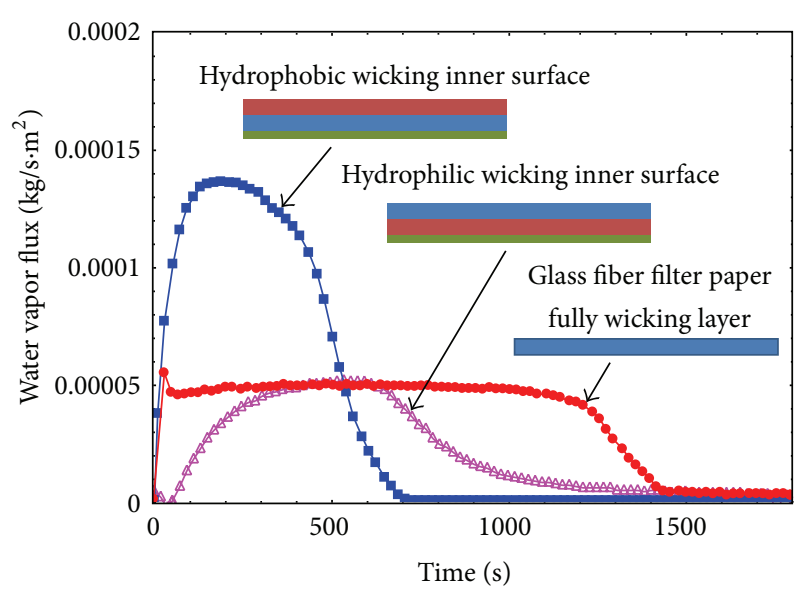

FIGURE 9: Water vapor transport from outer surface of fabric, following $0.1 \mathrm{~g}$ application of liquid water on inner surface of fabric. Sweep flow of $40 \%$ relative humidity on both sides of the fabric.

than is in the case for the laminate with the hydrophilic inner surface. The laminate with the hydrophilic inner surface transported about half the total amount of water as either the glass fiber filter or the laminate with the hydrophobic wicking inner surface. It is also interesting to note that although the membrane laminates showed poor water diffusion rates in the vapor diffusion test method, the transport behavior when the membranes are exposed to liquid water is much more efficient.

This can be further illustrated by looking at liquid water vapor transport rates when tested under a humidity gradient, as shown in Figure 10.
In this case, the fabrics are first equilibrated with a humidity of $0.8(80 \%$ r.h.) over the top of the sample, and 0.4 (40\% r.h.) across the bottom of the sample. Under these vapor transport conditions, the water vapor transport rate, of the reactive polymer membrane laminates are quite low and are an order of magnitude less than the water vapor transport rate through the reference glass fiber filter layer. This is also reflected in the water vapor transport curves presented previously in Figure 7, where the reactive polymer membrane laminates were shown to have much higher water vapor diffusion resistances than the commercial waterproofbreathable membrane laminates at all humidity conditions.

However, when the liquid water is applied to the inner comfort layer of the fabric, the water vapor transport rates increase in a similar fashion as was seen in Figure 9. Under these gradient conditions, the hydrophobic wicking layer is more successful at moving liquid water into the polymer membrane layer, increasing the water vapor transport rate quickly, and efficiently transferring water from one side of the laminate to the other. However, the water vapor transport rate does not exceed the performance of the reference glass fiber filter layer in this case but does achieve levels that are equivalent to the steady-state vapor transport rate of the glass fiber filter. The laminate with the hydrophilic wicking inner surface again lags behind the performance of hydrophobic wicking inner surface configuration because the liquid water is held away from the membrane. However, since the humidity in the flow in that upper portion of the cell is at $0.80(80 \%$ r.h.) evaporative losses due to the ventilating flow are much less, and there is more time for the liquid water to be taken up by the polymer layer and transported to the other side. Consequently, the total amount of water transported through the membrane is equivalent for the two laminates. It just takes a longer time for the same amount of water to 


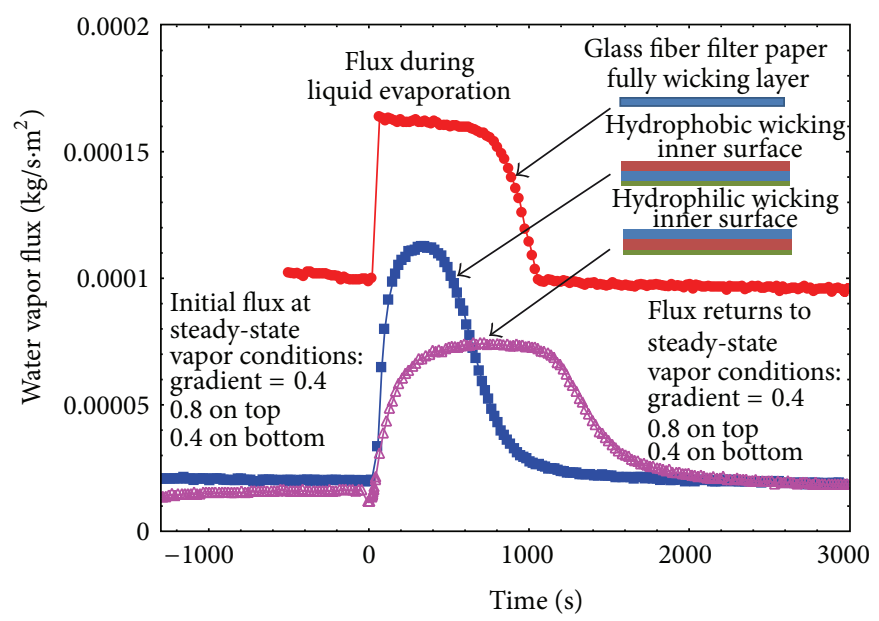

FIGURE 10: Water vapor transport under a humidity gradient of 0.4 ( 0.8 on top of the sample and 0.4 on the bottom of the sample).

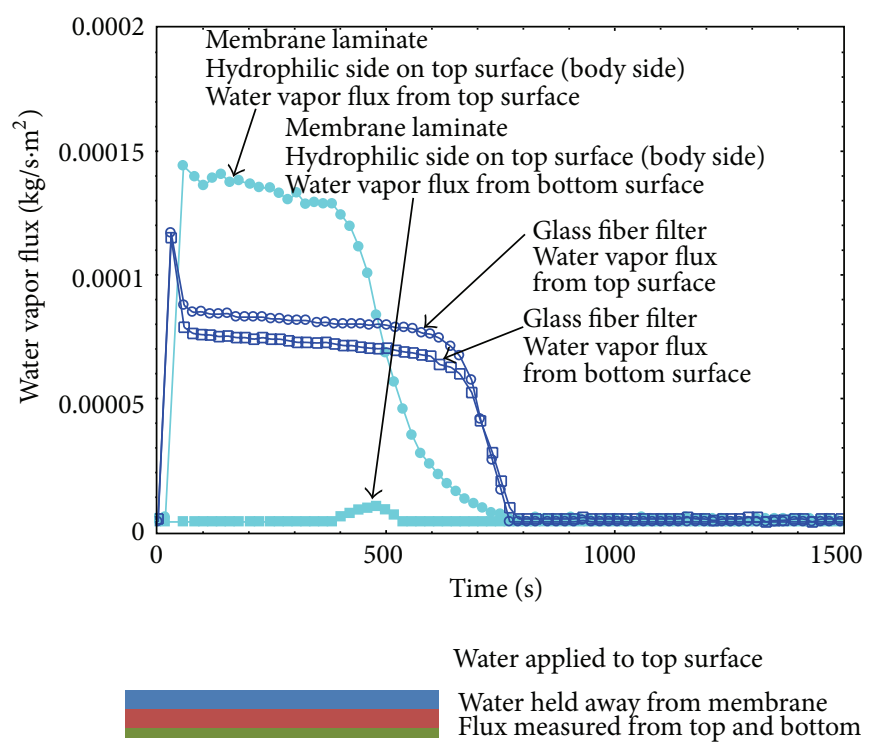

FIGURE 11: Water vapor transport from outer surface of fabric, following $0.1 \mathrm{~g}$ application of liquid water on inner surface of fabric. Sweep flow of $0 \%$ relative humidity on both sides of the fabric.

be transported through the laminate with the hydrophilic wicking inner surface.

The dual flow permeation test method also allows the directionality of water vapor permeation to be easily measured. Test conditions from Table 1 that use the same humidity on both sides of the sample should produce equivalent evaporation rates from a fully wicking sample. Both sides of the sample are wetted, and water evaporates equally well from both sides. However, we would expect that the reactive membrane laminate with the hydrophilic inner surface would have a very different behavior. The liquid water would be held in the hydrophilic layer and would mostly evaporate in the upper ventilating air flow, with much less diffusing through the polymer membrane to the other side of the permeation cell.
A good illustration of this directional permeation is obtained at the test condition of $0 \%$ humidity on both sides of the sample. This maximizes evaporation on the inner (upper side) of the test sample. Figure 11 shows that the glass fiber filter has equivalent water vapor flux from the top and bottom surfaces of the sample and that they both dry at the same rate.

The behavior of the laminate with the hydrophilic side towards the skin is dramatically different (Figure 11). Most of the liquid water is evaporated into the upper air flow. Only a tiny amount permeates through the polymer film, and only after a significant delay, and after most of the liquid water has already evaporated into the upper ventilating flow. It should also be noted that the polymer film is fully equilibrated to the humidity conditions of $0 \%$ r.h and contains very little water, 
resulting in a very low water vapor transport rate to begin with.

As in the previous examples, Figure 11 shows that when the hydrophilic layer is oriented towards the body, the layer fails to hydrate the polymer membrane effectively, and water vapor transport through the membrane is hindered.

Figures 9-11 illustrate the most important phenomena associated with the hydrophilic or hydrophobic orientation of the comfort layer on the reactive polymer membrane laminate. Other test conditions from Table 1 are consistent with these examples and support these general guidelines.

\section{Conclusions}

Permeation cell measurements demonstrated that the rate of liquid wicking was conveniently reflected in a measurable cooling effect on the fabric surface due to water evaporation. Results from vapor transport testing with wetting/nonwetting fabrics show that there are very large differences in drying time that can impact the rate at which the evaporating sweat from the body is transported through the fabric to the environment. Orienting a hydrophobic/hydrophilic fabric with the hydrophobic side towards the human skin surface and the hydrophilic surface towards a laminated reactive polymer membrane laminate is more efficient than the reverse orientation at picking up liquid from the skin surface and moving it to the polymer layer where it is available for hydration and chemical reaction.

\section{Conflict of Interests}

One of the authors, Dr. H. Benaddi, is the principal research scientist for the commercial entity, Stedfast, Inc., Canada, which manufactures the commercial material mentioned in the paper (Stedfast ChemCAT).

\section{References}

[1] S. Narang and S. Ventura, "Immobilized 4-dialkylaminopyridines: supernucleophilic chemical decontaminating agents," US Army Research Office Technical Report 23208.1-CH, 1987, http://handle.dtic.mil/100.2/ADA190999.

[2] S. Narang, S. Ventura, and R. Ramharack, "Polymeric dialkylaminopyridines as dupernucleophilic catalysts," in Chemical Reactions on Polymers, vol. 364 of ACS Symposium Series, chapter 6, pp. 72-80, 1988.

[3] S. Wang, L. Bromberg, H. Schreuder-Gibson, and T. Hatton, "Catalysis of organophophorous ester degradation by chromium (III) terephthlatalate (MIL-101) metal organic framework chelated to $N, N$-dimethylaminopyridine and related aminopyridines," ACS Applied Materials and Interfaces, vol. 5, no. 4, pp. 1269-1278, 2013.

[4] P. Gibson and H. Schreuder-Gibson, "Influence of hydration state on permeation testing and vapor transport properties of protective clothing layers," Journal of Engineered Fibers and Fabrics, vol. 4, no. 4b, pp. 11-20, 2009.

[5] J. Andzelm, J. Walker, H. Schreuder-Gibson, and P. Gibson, "Interaction of water and DFP with PVA-Am/PEI polymer blends," in Proceedings of the Chemical and Biological Defense
Physical Science and Technology Conference, Dallas, Tex, USA, November 2009.

[6] H. Schreuder-Gibson, J. Walker, J. Owens et al., "The role of water in the deactivation of chemical and biological agents within fabrics," in Proceedings of the Chemical and Biological Defense Physical Science and Technology Conference, New Orleans, La, USA, November 2008.

[7] P. Gibson, "Water-repellent treatment on military uniform fabrics: physiological and comfort implications," Journal of Industrial Textiles, vol. 38, no. 1, pp. 43-54, 2008.

[8] P. Dolhan, "A comparison of apparatus used to measure water vapour resistance," Journal of Coated Fabrics, vol. 17, pp. 96-109, 1987.

[9] B. Farnworth, W. Lotens, and P. Wittgen, "Variation of water vapor resistance of microporous and hydrophilic films with relative humidity," Textile Research Journal, vol. 60, no. 1, pp. 5053, 1990.

[10] P. Gibson, C. Kendrick, D. Rivin, M. Charmchi, and L. Sicuranza, "An automated water vapor diffusion test method for fabrics laminates, and films," Journal of Coated Fabrics, vol. 24, pp. 322-345, 1995.

[11] P. W. Gibson, "Water vapor transport and gas flow properties of textiles, polymer membranes and fabric laminates," Journal of Coated Fabrics, vol. 28, pp. 300-327, 1999.

[12] P. Gibson, D. Rivin, A. Berezin, and A. Nadezhdinskii, "Measurement of water vapor diffusion through polymer films and fabric/membrane laminates using a diode laser spectroscope," Polymer-Plastics Technology and Engineering, vol. 38, no. 2, pp. 221-239, 1999.

[13] P. W. Gibson, "Effect of temperature on water vapor transport through polymer membrane laminates," Polymer Testing, vol. 19, no. 6, pp. 673-691, 2000.

[14] D. Rivin, C. Kendrick, P. Gibson, and N. Schneider, "Studies of the Solubility and Transport Behavior of Water and Alcohols in Nafion," Polymer, vol. 81, p. 541, 1999.

[15] E. A. McCullough, M. Kwon, and H. Shim, "A comparison of standard methods for measuring water vapour premeability of fabrics," Measurement Science and Technology, vol. 14, no. 8, pp. 1402-1408, 2003.

[16] G. R. Lomax, "Breathable polyurethane membranes for textile and related industries," Journal of Materials Chemistry, vol. 17, no. 27, pp. 2775-2784, 2007. 

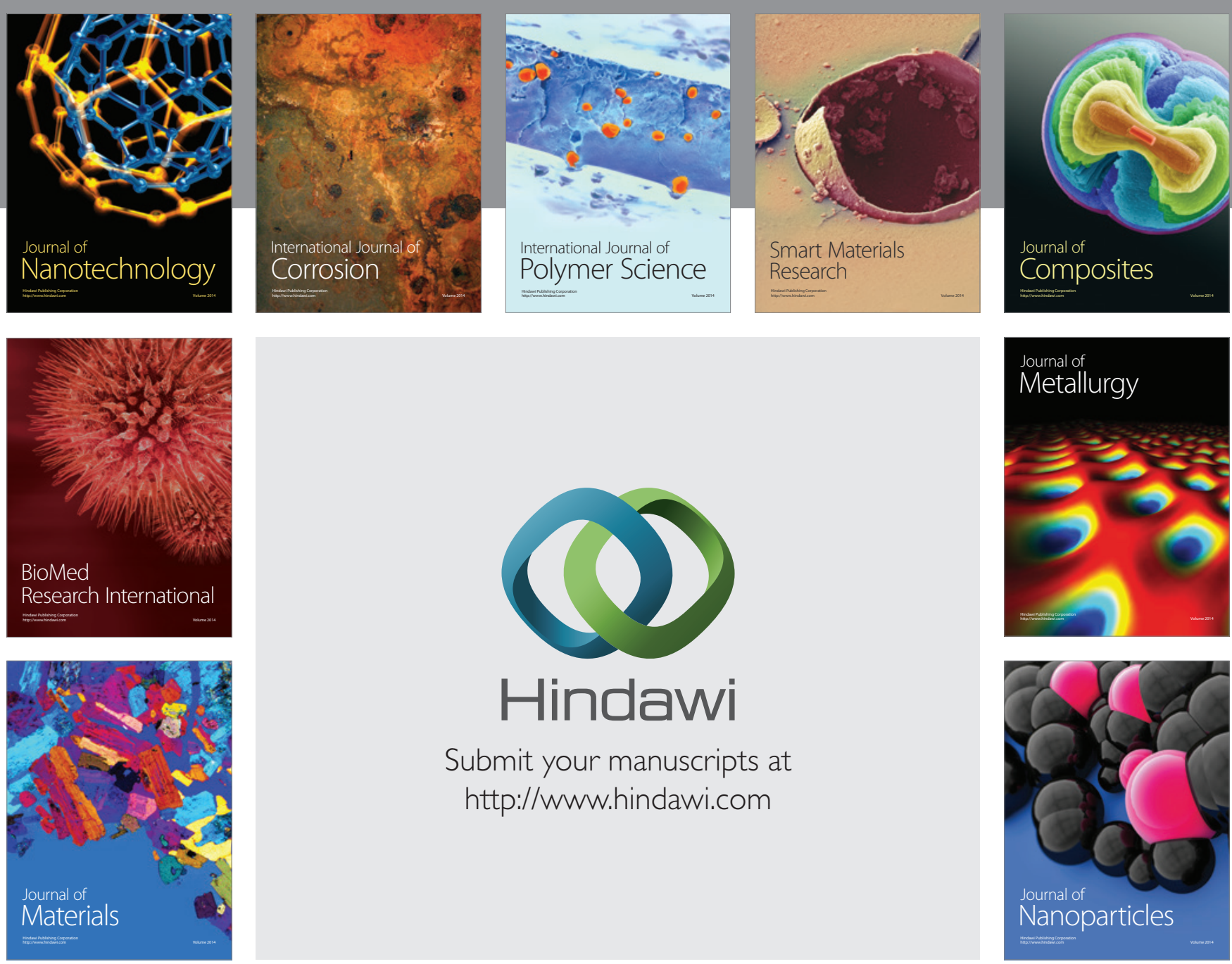

Submit your manuscripts at http://www.hindawi.com
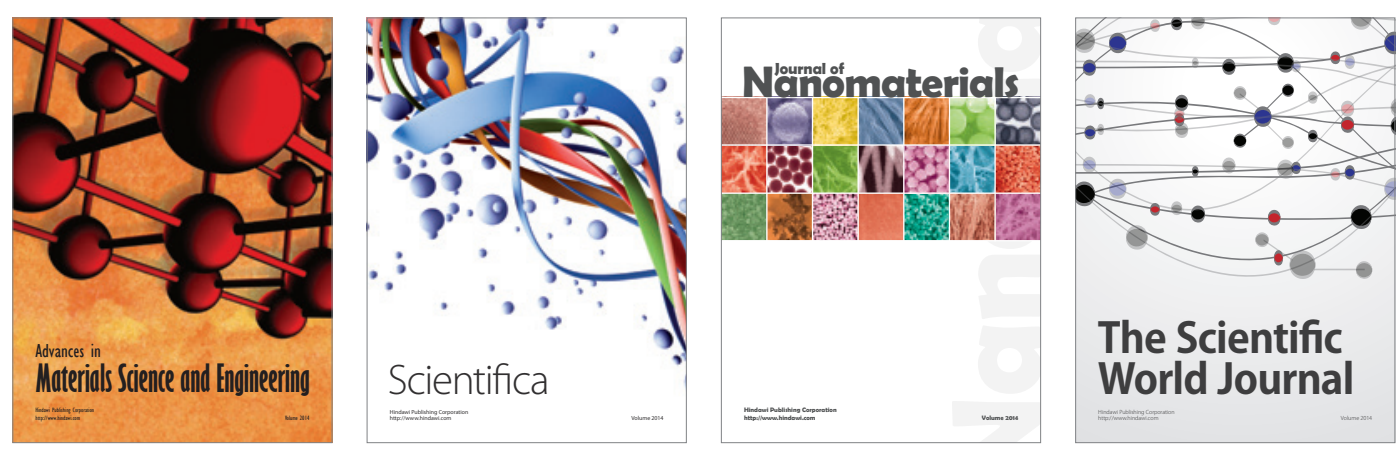

\section{The Scientific World Journal}
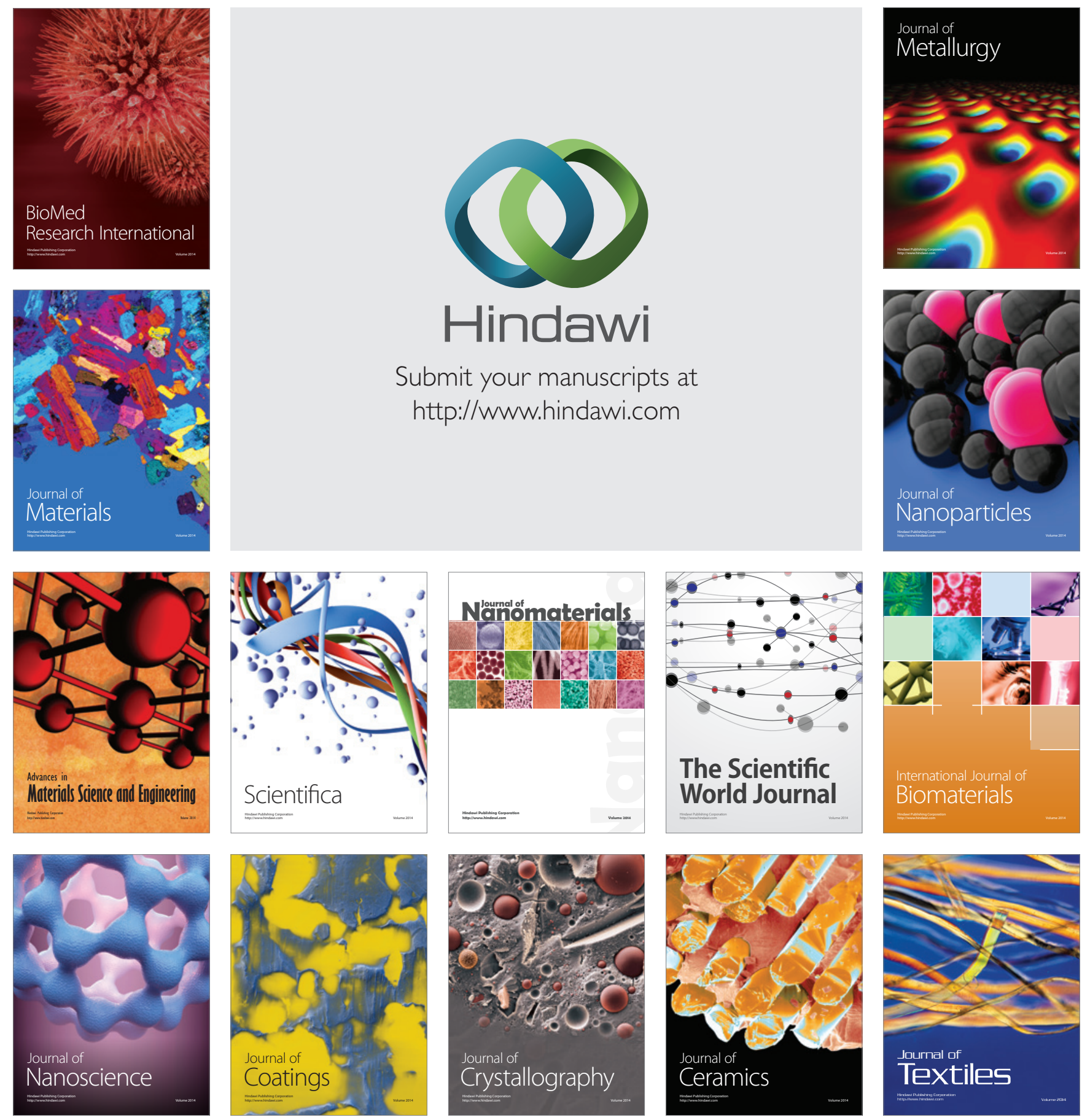